\title{
MOROCCAN DARIJA IN ONLINE CREATION COMMUNITIES: EXAMPLE OF WIKIPEDIA
}

\author{
Anass SEDRATI* \& Abderrahman AIT ALI*
}

BIBLID [1133-8571] 26 (2019) 11.1-14.

Resumen: «Dariya marroquí en las comunidades de creación en línea: ejemplo de Wikipedia». Las comunidades de creación en línea - CCL, son redes de personas que colaboran a través de plataformas de Internet para compartir y difundir conocimientos y contenido sobre diversos temas. Wikipedia es una de las ampliamente utilizadas CLL en el mundo, y tiene la misión de difundir todo el conocimiento humano a todas las personas en el globo. Uno de los pilares de Wikipedia es que todos los humanos deberían poder acceder a la información en sus propios idiomas. Una particularidad de este sistema es su descentralización e independencia de cualquier estado o autoridad, lo que permite a cualquier usuario ser parte de la comunidad y participar directamente para enriquecer los proyectos preferidos. En este contexto, este artículo presenta los proyectos existentes con el objetivo de proporcionar un contenido enciclopédico en dariya marroquí, como es el caso del proyecto de Wikipedia en esta lengua. En este artículo se presenta este proyecto, así como los diferentes desafíos a los que se enfrenta. Concluimos este artículo analizando las perspectivas futuras de la Wikipedia en Dariya y sugiriendo una serie de mejoras y desarrollos a seguir.

Palabras clave: Dariya, Marruecos, Creación en línea, Wikipedia, Internet, Descentralización.

Abstract: Online creation communities - OCCs, are networks of individuals who collaborate via internet platforms in order to share and spread knowledge and content on various topics. Among the largely used OCCs in the internet, we find Wikipedia which has a mission to spread the sum of human knowledge to all people in the world. One of the pillars of Wikipedia is that all humans should be able to access information in their own languages. A particularity of this system is its decentralization and independence from any state or authority, allowing any user to be part of the community and participate directly to enrich the projects of their choice. In this context, this paper

* PhD student at INPT, Rabat, Morocco, project Coordinator at Wikimedia Morocco User Group.

* Wikimedia Morocco, PhD student at KTH, Stockholm, Sweden. 
presents the existing projects aiming to provide an encyclopedic content in Moroccan Darija such as Wikipedia in Darija project. We discuss their status as well as the different challenges facing them. We conclude this paper by analyzing the future prospects of these projects and by suggesting a number of improvements and developments to follow.

Key words: Darija, Morocco, Online Creation, Wikipedia, Internet, Decentralization.

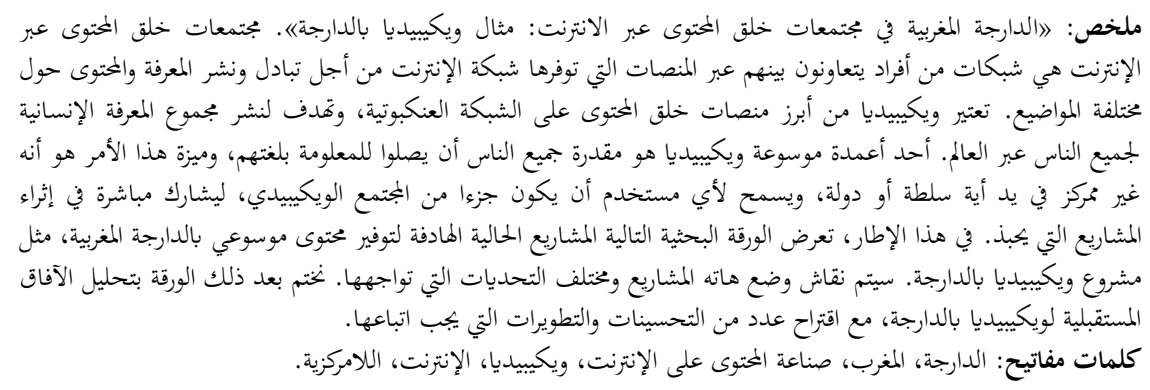

\section{Introduction}

The internet has long been defined as a network of computer networks. This definition was adopted since 1990 when Tim Berners-Lee, an English engineer and computer scientist at CERN, invented the Web or what is commonly known as the World Wide Web - WWW (McPherson 2009: 5). In these early days, the internet was mostly accessible using web browsers and the content of the web pages was made available by the creators of the websites. Users could only navigate the content from one web page to another using hyperlinks. The internet online content was largely provided by website publishers and rarely users.

In the last years, creation of online content has changed considerably and is significantly different than what it was at the creation of the internet. Internet users around the world are now contributing to the creation of large quantities of information and digital content as never before. In contrast to the old WWW, Web 2.0 is commonly referred to as the participative, participatory or social Web (O'Reilly 2009: 2). In this new version, users can create contents in various forms such as through social networking sites (e.g. Facebook, Twitter), blogs, wikis (e.g. Wikipedia) and video sharing sites (e.g. YouTube).

Wikipedia is one of these large platforms where creating online content users, based on wikis, are organized in various interconnected communities 
depending on their topics of interest and languages, with the aim of spreading the sum of human knowledge in the form of a large online encyclopedia. The latter shall be freely available to anyone, anywhere and in as many languages as possible.

Moroccan Darija is generally underrepresented online and has a very limited quantity of online content. A Darija version of Wikipedia could mean an increase representation of online content in Darija and hence the beginning of a quality presence of Darija online.

This paper aims at investigating the online content creation in Darija through Wikipedia in particular. It also discusses the challenges and issues that are faced by the projects and initiatives promoting online creation of contents in Moroccan Darija.

The paper is structured as follows: Section 1 provides some background information about online creation of contents and their communities as well as a zoom into Wikipedia and its communities before describing the situation of Darija online. Section 2 presents the online creation communities in Morocco and highlights Wikipedia in Morocco and in Darija before explaining the different challenges that are faced by these communities. Conclusions are provided in section 3 that ends the paper.

\section{Background}

This section gives background information and overview on relevant topics. We start by explaining OCCs in general and then the particular case of Wikipedia. We conclude this section by briefly presenting the situation of OCCs in Moroccan Arabic.

\subsection{Online creation communities}

OCCs, short for online creation communities, are internet-based virtual communities whose members interact and collaborate via the internet in order to create online contents (Fuster Morell 2010: 7). They create these contents on different topics and using various languages and formats. International communities create online content in widely spoken languages such as English, Mandarin, Spanish and French. However, content in minority languages is mostly produced by communities whose members belong to a local community such as the Moroccan online community. 
Until recently, most of the content that was produced and published online was mainly through traditional chat rooms, forums and blogs, to name few examples. However, OCCs initiated a new era where the created content is freely accessible, editable and owned by anyone ${ }^{(1)}$. The ultimate aim is sharing common information resources resulting from collaboratively «building and integrating dispersed information and knowledge» (Fuster Morell 2010: 7).

\subsection{Wikipedia - Wikimedia presentation}

Wikipedia is a multilingual collaborative free-content online encyclopedia based on the model of openly editable content, i.e. wiki. Both Wikipedia source code and content are in fact open and free and can be edited by everyone. It was created in January 2001 by Jimmy Wales and Larry Sanger to «provide free knowledge for all»(2). This encyclopedia is among the most visited websites worldwide, currently ranked $\operatorname{sixth}^{(3)}$. Wikipedia is available in more than 300 languages (304 as of July 2019) that gather in total more than 40 million articles. In each language, the corresponding Wikipedia is independent and has its own community, responsible for making rules and enforcing policies related to that language edition. Wikipedia is just one of 12 projects managed by the Wikimedia Foundation. The complete list of these projects can be found at the official web page of the foundation ${ }^{(4)}$.

The Wikimedia movement is the global community of contributors to the Wikimedia Foundation projects. One of the main aims behind the existence of this movement is to coordinate and organize how the community is working. It allows also activities to happen offline, and to support contributors worldwide through several ways (e.g. capacity building, sharing experience, etc.). Through the Wikimedia movement, users belonging to the same community can create and gather into different organizations such as chapters and local or thematic user groups.

(1) Ismael Peña-López, Citizen politics (VI): Online Public Sphere, ICTlogy, 2009 <https://ictlogy.net/20090530-citizen-politics-vi-online-public-sphere/> [31 July 2019].

(2) Wikipedia page about Wikipedia, $2019<$ https://en.wikipedia.org/wiki/Wikipedia > [31 July 2019].

(3) Alexa ranking of most visited websites worldwide, Wikipedia 6th, Alexa, 2019 <https://www.alexa.com/topsites> [31 July 2019].

(4) List of all Wikimedia Foundation projects, Wikimedia Meta, 2019 <https://meta.wikimedia.org/wiki/Wikimedia_projects> [31 July 2019)]. 
Given its extent, size and influence, Wikipedia can logically be considered as a reference to analyze when studying Online Creation Communities. Hence its choice to be an example for an OCC in the present paper.

\subsection{Moroccan Darija online}

Situated at a crossroad between Africa, Europe and the Islamic world, Morocco has historically been a region where different cultures met and mixed with each other. An interesting outcome of this rich history was the emergence of the so-called Moroccan Darija, a combination of Arabic and Tamazight influenced by several foreign languages such as French and Spanish (Chafik 1999: 8). Formally, there are two official languages in Morocco: Arabic and Tamazight ${ }^{(5)}$. In practice however, it is not clear which Arabic or Tamazight versions are the official ones. The constitution does not mention if the official language is classical Arabic or Darija, but the common practice is that written documents are in classical Arabic (and/or French). Classical Arabic and more recently standard Tamazight are languages that are taught since the first year of school, while daily life oral communication happens mostly in Darija and/or Tamazight. There is no official version of written Darija, and it is not part of any program in the official education curriculum. Written poems, magazines and even books exist in Darija (Elinson 2013, Miller 2017). However, the lack of official standardization makes the adoption and broadening of any of the chosen ways difficult and conflictual. Moreover, the social status of writing in Darija is perceived as far lower and devaluing compared to a similar text in the languages of instruction in the Moroccan school: Classical Arabic or French (Zouhir 2014). Writing in Darija can be interpreted either as a lack of mastering of the «formal» languages, or as an act of rebellion against them. Either way, this choice is interpreted negatively by several actors ${ }^{(6)}$.

(5) English translation of Morocco's 2011 constitution

<https://www.constituteproject.org/constitution/Morocco_2011.pdf?lang=en> [31 July 2019].

(6) Abdellah Laroui: «Darija level is only limited to communication» [in Arabic], Alyaoum24,

2013 <http://www.alyaoum24.com/167927.html> [31 July 2019]. Moussa Chami (Moroccan

Association for defending Arabic): «Darija does not have any scientific specifications to be

the language of writing science» [in Arabic], Attajdid, 2009

<https://www.maghress.com/attajdid/49934> [31 July 2019]. 
With the introduction of the internet in Morocco and its boom in the 21st century, several voices started to challenge the official view regarding languages hierarchy in Morocco. The spreading of the internet and its related freedom within the younger generation allowed them to choose to communicate in the language of their choice, without any restriction or need for standardization. An example of this wave of challenging voices are Farouk El Merrakchi ${ }^{(7)}$ Mourad Alami $^{(8)}$, or Hamid El Mahdaoui (Sedrati 2017). Using Moroccan Darija in communication is not perceived in the same way depending on its form and way of use. If oral Darija is widely accepted and is even present in official channels, its written version is constantly criticized for several reasons ${ }^{(9)}$ (Hoogland 2018). First, a problem when writing in Darija is its non-standardization, allowing each and every one to write the way they want (Hall 2015). Related to this argument comes the alphabet problem. Since the first keyboards introduced to Morocco were in Latin Alphabet, a version of Darija is written in that Alphabet (for practical reasons), while neo-Darijists tend to prefer the Arabic alphabet. Second, writing in Darija is perceived by some as a distortion of the Arabic language itself. The eternal debate of the differences between languages and dialects emerges at that point (Haugen 1966). It is worth to mention that several attempts for a standardized Darija alphabet exist, interested readers are referred to (Aguadé 2006), (Hoogland 2014) and (Moustaoui 2012).

\section{OCC in Moroccan Darija}

As presented earlier, Wikipedia is a reference example for an OCC. Studying users' behavior in Wikipedia will give insights about OCCs. This section focuses on the situation of Wikipedia in Morocco, Darija version of the encyclopedia as well as its challenges and issues.

(7) Farouk El Merrakchi, «writer of the first scientific books in Darija» [in French], Medias24, 2013 <https://www.medias24.com/SOCIETE/6642-Une-premiere-Un-Marocain-redige-desmanuels-scientifiques-en-darija.html> [31 July 2019].

(8) Mourad Alami: «Besides being oral, Darija must also become written» [in French], Yabiladi, 2011 <https://www.yabiladi.com/articles/details/4144/mourad-alami-plus-etre-orale.html> [31 July 2019].

(9) Sarra GDR: «Arabizi: where numbers become letters», Medium, 2017 <https://medium.com/reputation-squad/arabizi-where-numbers-become-letters 635467996111> [31 July 2019]. 


\subsection{Wikipedia in Morocco}

The official representation of the Wikimedia Foundation in Morocco comes through the Wikimedia MA User Group ${ }^{(10)}$. The Moroccan user group was created in October 2015 to contribute in the advancement of the free knowledge movement in Morocco. Among the activities of the user group, one can mention the following: a) improving Wikipedia content related to Morocco, and b) empowering the local community in order to encourage local content in local languages.

In this context, these activities are not only related to content, but also to languages. The Wikimedia vision ${ }^{(11)}$ stresses on the importance of «providing all human beings with the sum of human knowledge». To complete this vision, it is therefore important to reach everyone by making content available in all languages. The Moroccan user group serves in that sense the interests of the Moroccan community, by making sure that the content is available not only in the official languages, but in all forms of expression that are present in Morocco, including Darija, and all Amazigh dialects.

In opposition to a formal institution, the user group is community driven. This setup allows the interests of the community to be respected and reflected in the group work. It is one of the strengths of the Wikimedia model. It is natural that conflicting views exist in the community but having a Wikipedia in Darija does not contradict having another one in classical Arabic. In fact, those interested in writing in Darija would either write in it or not write at all. The two projects are independent and are hosted on different platforms and pages. Moreover, the idea of providing knowledge to all is against any kind of discrimination towards a mode of expression and should empower people speaking given languages to write and read knowledge in their languages as well.

(10) Wikimedia Morocco User Group, Wikimedia Meta, 2019, <https://meta.wikimedia.org/wiki/Wikimedia_MA_User_Group > [31 July 2019]

(11) Wikimedia vision, Wikimedia Foundation, 2019 <https://wikimediafoundation.org/about/vision/ > [31 July 2019]. 


\subsection{Wikipedia in Moroccan Darija}

A good example of a project in Moroccan Darija by an OCC is the Moroccan Arabic version of Wikipedia ${ }^{(12)}$. The main concept is to have a version of the largest online encyclopedia also in Darija. This project is at the incubator stage, which is an early stage where any Wikimedia project starts before becoming an official project. In fact, an example of an official Wikipedia in an Arabic dialect is the Egyptian Arabic version of Wikipedia which has reached more than 20000 articles $^{(13)}$.

Similar to the Egyptian version, the aim of a Wikipedia in Darija is to make knowledge available online for people in Darija. This gives the Moroccan online community a relative independence from Eastern (in Classical Arabic) and Western (French, Spanish and English) shaped online contents, which often reflect poor knowledge about areas such as the local Moroccan culture or history. Thus, with more awareness of the local Moroccan specificities, in Darija it will be possible to produce and make available knowledge that is more extensive, more accurate and more accessible.

\subsection{Challenges and issues of Wikipedia in Darija}

Writing a version of the largest online encyclopedia in Moroccan Darija is not an easy task and has various and multiple challenges and issues. Among these, we find the following:

\subsubsection{Standardization}

Wikipedia is a written encyclopedia and most of its content is in written form, with a noticeable presence of images, audio and video files. On the other hand, Darija is an oral heritage that was never officially standardized in a written form. The difference of local variations in Darija itself, as well as the choice of different vocalizations leads to a pseudo-anarchical situation within

(12) Wikipedia in Moroccan Darija, Home Page, Wikimedia Incubator, 2019 <https://incubator.wikimedia.org/wiki/Wp/ary/\%D8\%A7\%D9\%84\%D8\%B5\%D9\%81\%D8 \%AD\%D8\%A9_\%D8\%A7\%D9\%84\%D8\%B1\%D8\%A6\%D9\%8A\%D8\%B3\%D9\%8A\%D8 $\%$ A9 > [31 July 2019].

(13) Wikipedia in Egyptian, Home Page, Wikipedia, 2019 <https://arz.wikipedia.org/wiki/\%D8\%A7\%D9\%84\%D8\%B5\%D9\%81\%D8\%AD\%D9\%87_ \%D8\%A7\%D9\%84\%D8\%B1\%D8\%A6\%D9\%8A\%D8\%B3\%D9\%8A\%D9\%87 > [31 July 2019]. 
the incubator. Some users insist on using their own regional dialect based on the principle of «write Darija the way what you speak it», which creates conflicts between the different (and few) active users. Even if speaking the exact same dialect, two different users can use different alphabets, or different letters to vocalize their words. For example, the same verb, say, can be written in different ways as presented in Table 1 . The writing depends on the factors mentioned above. Creative users can even go further and write the same sounds in Tifinagh alphabet, claiming that it is an alphabet suitable for Darija as well.

\begin{tabular}{|c|c|c|c|}
\hline \multirow{2}{*}{$\begin{array}{l}\text { Verb } \\
\text { (English) }\end{array}$} & \multirow{2}{*}{$\begin{array}{l}\text { Classical } \\
\text { Arabic }\end{array}$} & \multicolumn{2}{|r|}{ Darija } \\
\hline & & $\begin{array}{l}\text { Arabic } \\
\text { alphabet }\end{array}$ & $\begin{array}{l}\text { Latin alphabet and } \\
\text { numbers }\end{array}$ \\
\hline $\begin{array}{l}\text { Say } \\
\text { (imperative) }\end{array}$ & قل & 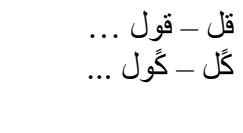 & $\begin{array}{l}\text { qol, qoul, qul, ... } \\
\text { 9ol, 9ul, 9ol, 9oul, ... } \\
\text { gol, gul, goul, ... }\end{array}$ \\
\hline
\end{tabular}

Table 1 - Example of different writing for the verb «say».

\subsubsection{Keyboards}

One direct consequence of the non-standardization of Darija is the splitting happening in the choice of the writing system. The background of this issue goes back to the first computers entering Morocco, that had only Latin letters in their keyboards. This obliged many people to «adapt» and write their language in this available alphabet. Nowadays, the situation has changed and keyboards with Arabic letters are widely present in the country. Nonetheless, the issue of which alphabet to use still remains for different reasons. For one, some are used to the Latin alphabet as it was the first alphabet which they used to write Darija and are now comfortable with it. Second, many Moroccan born outside of Morocco (mainly in Europe) do not know the Arabic alphabet and their only possibility to write in Darija is to use the Latin letters. Meanwhile, most articles in Moroccan Wikipedia are in Arabic letters, as it is the way active users have chosen to continue with. A summary of the keyboard issues and an attempt to solve it was proposed by the two Moroccan Wikipedians Nassim Regragui and 
Tariq Daouda in their initiative «Ktb Darija» ${ }^{(14)}$. Examples of writings in Darija can be found in the presentation slides from a conference organized by Wikimedia Morocco in Rabat in $2016^{(15)}$.

\subsubsection{Vulnerability}

Given its small size and relative low activity as of April 2019, the Darija Wikipedia in the incubator is very vulnerable and suffers from recurrent vandalism by those refusing a given alphabet, a specific dialect, or even questioning the existence of Darija Wikipedia itself. The talk page of Darija in the incubator ${ }^{(16)}$ gives an example of such criticism. If administrators are absent for a long period from the project, the whole Wikipedia (currently 136 articles, July 2019) can easily be vandalized. This situation discourages motivated editors, who can see their efforts vandalized and destroyed by different kinds of opponents.

\subsubsection{Interest}

Written Darija does not have an official status and has never been recognized at any level in the state. Usually, Moroccans able to read and write can first write in Classical Arabic, and (lower percentage) in French. One of the main arguments used by opponents to the Darija project is that those who can read Darija can by definition read, and therefore can also read (at least) Classical Arabic and eventually French. So why is this version existing as it seems to be superfluous?

The answer can naturally be found in the Wikimedia vision, stating that knowledge shall be available in all languages, and that people have freedom to choose the language they want to read in. Otherwise, one can claim that since science is produced in English, all those knowing how to write and read in English do not need to translate knowledge to their languages as it is a «waste»

(14) Ktb Darija, iniatiative to create a standardized written form of Darija, 2019 <https://ktbdarija.com/> [31 July 2019].

(15) Slides presenting Wikimedia and the User Group in Moroccan Darija, Wikimedia Morocco, 2016 < https://meta.wikimedia.org/wiki/Presentations/ary > [31 July 2019].

(16) Talk Page related to the Darija project, Wikimedia incubator, 2019 <https://incubator.wikimedia.org/wiki/Talk:Wp/ary/\%D8\%A7\%D9\%84\%D8\%B5\%D9\%81 \%D8\%AD\%D8\%A9_\%D8\%A7\%D9\%84\%D8\%B1\%D8\%A6\%D9\%8A\%D8\%B3\%D9\%8A $\%$ D8\% A9 > [31 July 2019]. 
of time and resources. Moreover, not all the Moroccans speak a «school» language or are able to read it. Since Wikipedia offers also audio and video support, having even articles about «basic» subjects such as the countries of the world and the different planets of the solar system in Darija is useful. People can indeed use audio support to listen to the articles and understand them, unlike Classical Arabic or French that are not understood by all the Moroccan population.

\subsubsection{Community}

The Darija Wikipedia community is very scattered, with users living in different sides of the globe, and barely communicating with each other. There are no meetings (either online or physical) to synchronize and align regarding the challenges above, especially ones related to standardization and writing conventions. A number of users prefer to edit articles and not discuss their choices, making the Darija incubator experimenting an enormous variation from one article to the other, either in terms of dialectal differences, or in terms of form (alphabet, choice of standard).

Most of the editors are only active online, sometimes with anonymous usernames, which makes the coordination and consensus reaching very challenging. Moreover, and even if the community is relatively small, ideologies and angles of proceeding are widely different, which jeopardizes efforts of collaborations to move on with the content from the incubator towards a full and complete Wikipedia version.

\section{Conclusions}

Internet has proven to be a useful platform to empower various ways of expression given its free nature and absence of grammatical or lexical controls. In this context, the use of Darija has grown online and it is not unusual to notice Facebook pages and electronic texts written in one of the different Darija writing forms. The focus of the present paper was to investigate the presence of Darija in Online Creation Communities (OCC), through the example of Wikipedia, the biggest online encyclopedia in the world. As an open platform, Wikipedia allows users to produce and write knowledge in all languages. The latter encourages developing content in Darija, first to guarantee the presence of this way of expression online, but also to guarantee a relative independence to 
the Moroccan content, often shaped by Eastern (Classical Arabic) or Western (French, English) users.

Currently, Darija Wikipedia in the incubator faces a number of challenges, related mostly to the low number of active users, and to the general status of not-standardized writing system for it, that reflects on the encyclopedia as well. However, including more editors to participate in the effort of improving the Darija version of Wikipedia on a bigger extent will push to standardize it, or at least to adapt conventional standards for the encyclopedia. By being inclusive and allowing everyone to edit articles, Wikipedia guarantees in fact diversity and representability of different opinions and ideologies, and experiences from Wikipedias in other languages facing standardization challenges did all prove the possibility of converging towards successful solutions ${ }^{(17)}$. The Moroccan Darija Wikipedia can also benefit from the experience of the Algerian Darija sister project, which collaborated with national universities to improve the content quality, as well as to decide on the used Alphabet (Arabic letters). The Algerian Wikipedia counts nearly 1000 articles and can be worth reading ${ }^{(18)}$.

In summary, OCCs are an excellent platform to empower emerging languages and dialects online. Users are in fact free to express themselves in their language without being stopped or moderated. Moreover, people speaking these languages will be able to find quality enlightening content, that is fully understood by them, in their mother tongue. Darija in Wikipedia benefits Moroccan users who can have an independent encyclopedia made by Moroccans, but it benefits also Darija itself, by pushing it to be standardized through the convergence of the community discussions that are ongoing around the project. By writing this paper, the authors hope that a wider participation in the project will help solve the current challenges and make the Darija Wikipedia reach maturity and success.

$$
* * *
$$

(17) Wikipedias in multiple writing systems, Wikimedia Meta, 2019 <https://meta.wikimedia.org/wiki/Wikipedias_in_multiple_writing_systems > [31 July 2019].

(18) Wikipedia in Algerian Darija, Home Page, Wikimedia Incubator, 2019 <https://incubator.wikimedia.org/wiki/Wp/arq/\%D8\%A7\%D9\%84\%D9\%BE\%D8\%A7\%D8 \%AC\%D8\%A9_\%D8\%A7\%D9\%84\%D9\%84\%D9\%88\%D9\%84\%D8\%A7\%D9\%86\%D9\% 8A\%D9\%91\%D8\%A9 > [31 July 2019]. 


\section{References}

AGUADÉ, Jordi. 2006. «Writing dialect in Morocco». Estudios de Dialectología Norteafricana y Andalusí 10, pp. 253-274.

CHAFIK, Mohammed. 1999. Addarija Al Maghribiya: majalu tawarudin bayna alamazighija wal3arabiya [in Arabic]. Rabat, Academy of the Kingdom of Morocco.

ELINSON, Alexander. 2013. «Dārija and changing writing practices in Morocco». International Journal of Middle East Studies 45(4), pp. 715730.

FUSTER MORELL, Mayo. 2010. Governance of online creation communities: Provision of infrastructure for the building of digital commons [Doctoral dissertation]. Florence, Italy, European University Institute.

HALL, Jennifer Lee. 2015. Debating Darija: Language Ideology and the Written Representation of Moroccan Arabic in Morocco [Doctoral dissertation]. USA, University of Michigan.

HAUGEN, Einar. 1996. «Dialect, Language, Nation 1». American anthropologist 68, 4, pp. 922-935.

HOOGLAND, Jan. 2014. «Towards a standardized orthography of Moroccan Arabic based on best practices and common ground among a selection of authors». In: Árabe marroquí: de la oralidad a la enseñanza. Paula Santillán Grimm, Luis Miguel Pérez Cañada \& Francisco Moscoso García (eds.). Colección estudios 140. Cuenca, Universidad de Castilla-La Mancha, pp. 59-76.

HOOGLAND, Jan. 2018. «Darija in the Moroccan press: The case of the magazine Nichane». In: Sociolinguistic Studies 12, 2, pp. 273-293.

MCPHERSON, Stephanie Sammartino. 2009. Tim Berners-Lee: Inventor of the World Wide Web. Minneapolis, Twenty-First Century Books.

MILLER, Catherine. 2017. «Contemporary dārija Writings in Morocco. Ideology and Practices». In: The politics of written language in the Arab world. Jacob Høigilt \& Gunvor Mejdell (Eds.), Leiden, Brill, pp. 90-115.

MOUSTAOUI, Adil. 2012. «Language planning, standardization and dynamics of change in Moroccan Arabic». Dialectologia 9, pp. 53-69.

O'REILLY, Tim. 2009. What Is Web 2.0. Sebastopol, O'Reilly Media, Inc. 
SEDRATI, Anass. 2017. The use of YouTube in Morocco as an instrument of social critique and opposition: Three cases: Richard Azzouz, Hamid El Mahdaouy, Najib El Mokhtari [Bachelor Thesis]. Stockholm University.

ZOUHIR, Abderrahman. 2014. «Language policy and state in Morocco: The status of Berber». Digest of Middle East Studies 23, 1, pp. 37-53. 